\title{
Effect of Astaxanthin on cell viability in T-47D and MDA-MB-231 Breast Cancer Cell Lines
}

\author{
Aida Karimian ${ }^{1}$, Mohammad hadi Bahadori ${ }^{2}$, Akbar Hajizadeh \\ Moghaddam ${ }^{1}$, Fereshteh Mir Mohammadrezaei ${ }^{1, *}$ \\ ${ }^{1}$ Department of Biology, Faculty of Basic Sciences, University of Mazandaran, \\ Babolsar, Iran \\ ${ }^{2}$ Cellular and molecular research center, Faculty of Medicine, Guilan University of \\ Medical Sciences, Rasht, Iran \\ ${ }^{*}$ Corresponding author: Fereshteh Mir Mohammadrezaei, Department of Biology, \\ Faculty of Basic Sciences, University of Mazandaran, Babolsar, Iran, E-mail: \\ Fereshteh.mmrezaei@gmail.com
}

DOI: $10.21859 /$ mci-supp-71

\section{Keywords:}

Astaxanthin

Cell Viability

Apoptosis

T-47D

MDA-MB-231

\begin{abstract}
Introduction: Breast cancer is the second frequent malignancy after lung cancer in the world and the most common cancer among women. Breast cancer can be classified by level, pathology, rate, and expression of estrogen receptor (ER), progesterone receptor (PR), or human epidermal growth factor receptor (Her2/neu). T-47D cells are characterized as $\mathrm{ER}+, \mathrm{PR}+$, and Her2/neu positive/luminal mammary carcinoma, while MDA-MB-231 cells are characterized as ER, PR-, and Her2/neu negative/basal-B mammary carcinoma. Astaxanthin is a xanthophyll carotenoid and extracted from the green microalgae Haematococcus pluvialis, the red yeast Phaffia rhodozyma, and crustaceans such as crab, shrimp, and lobster. The properties of Astaxanthin are antioxidant, antitumoral, and anti-inflammatory. In this study, the apoptotic effects of Astaxanthin on breast cancer cell lines have been investigated.
\end{abstract}

Materials and Methods: The human breast cancer cell lines T-47D and MDA-MB-231 were cultured in DMEM medium with $10 \%$ fetal bovine serum (FBS), 1\% penicillin/ streptomycin in a humidified $5 \% \mathrm{CO} 2$ atmosphere at $37^{\circ} \mathrm{C}$. The cells were seeded in $96-$ well plates, then were treated with various concentrations of Astaxanthin (1, 10, 50, 100 $\mu \mathrm{M})$ for 24 hours. Cell viability was assessed by MTT assay. Apoptosis was detected by Hoechst 33342 staining. Results Results showed that Astaxanthin significantly decreased cell viability in T-47D and MDA-MB-231 cell lines in a dose-dependent manner after 24 hours $(p<0.05)$. The most significant effect of Astaxanthin was at a concentration of 100 $\mu \mathrm{M}$. Also, Hoechst 33342 staining assay showed that Astaxanthin increased apoptosis at a dose of $50 \mu \mathrm{M}$ in T-47D and MDA-MB-231 cells.

Conclusions: The research findings indicate that Astaxanthin can induce apoptosis in cancer cells and it could be considered as a therapeutic agent in breast cancer. 